\title{
Evaluation of Echocardiographic Findings of Mucopolysaccharidosis Cases
}

(1) Nafiye Emel Çakar ${ }^{1}$, (D) Meryem Karaca²

1istanbul Okmeydanı Training and Research Hospital, Clinic of Pediatric Metabolism, Istanbul, Turkey

${ }^{2}$ Harran University Faculty of Medicine, Department of Pediatric Metabolism, Şanlıurfa, Turkey

\section{Abstract}

Objective: Mucopolysaccharidosis (MPS) is a lysosomal storage disease in which the degradation of glycosaminoglycans is impaired. Cardiac involvement may occur in different ways in all types of the disease. In this study, we aimed to evaluate the echocardiographic reports of our patients with MPS retrospectively.

Methods: Echocardiography reports of 37 patients with MPS were reviewed retrospectively.

Results: Cardiac involvement was present in $70.2 \%$ our patients and the most commonly involved structure was mitral valve (59.5\%). The most common pathology in mitral valve was mitral valve regurgitation (51.4\%).

Conclusion: Cardiac involvement and complications are frequently seen in MPS. Heart failure, coronary artery involvement and arrhythmias are the main causes of death. Early diagnosis of MPS and early initiation of enzyme replacement therapy may improve cardiac involvement. Progressive valve involvement may require surgical intervention over time. For all these reasons, cardiac evaluation in MPS patients should be performed at least once a year with accompanying electrocardiogram and echocardiography.

Keywords: Mucopolysaccharidosis, echocardiography, cardiac involvement, mitral valve

\section{INTRODUCTION}

Mucopolysaccharidosis (MPS) are a group of lysosomal storage diseases characterized by chronic, progressive and multiple system involvement due to impaired glycosaminoglycans (GAG) degradation. There are 7 types of MPS as type I, II, III, IV, VI, VII and IX. MPS type III and IV are further sub-classified (1). There is wide clinical heterogeneity within this group of disorders, such as a dysmorphic features, learning difficulties, behavioral disturbance and bone dysplasia (2). Cardiac involvement can be seen in all types of MPS, and it is more common in MPS types I, II and VI, which have dermatan sulfate accumulation (3). Cardiac involvement may present with different presentations with the accumulation of GAGs in the cells of the endocardium, myocardium, valves, coronary arteries and transmission system. Cardiac findings, from asymptomatic valve involvement to severe heart failure, may be severe enough to cause mortality at an early age (3). The most common cardiac involvements in patients with MPS are thickening and deformity of the mitral and aortic valves, respectively (3). In this study, echocardiography reports of patients with MPS, who were diagnosed by enzymatic or genetic analysis, were examined and cardiac pathologies were evaluated according to MPS types.

\section{METHODS}

Patients diagnosed with MPS through enzymatic or genetic examinations in two centers were included in this study. Patients who did not attend follow up examinations in the last year or who did not undergo echocardiography in the last year were excluded from the study. Thirty-seven patients who fulfilled these conditions were included in the study and their files were reviewed retrospectively.

\section{Statistical Analysis}

All values were given as mean and standard deviation. Statistical 
analyzes were performed using SPSS 19 for Windows and Microsoft Excel 2017.

\section{RESULTS}

A total of 37 patients ( 15 female and 22 male) with MPS were included in the study. Four patients were MPS type 1, two were MPS type II, six were MPS type III, nine were MPS type IV and 16 were MPS type VI. The mean age of all patients was $99.9 \pm 67.5$ months. Gender distribution and mean ages according to MPS types are given in Table 1. MPS type II patients with X chromosome transmission were male and had the lowest mean age. The highest mean age of the patients was in MPS type III.

All echocardiography reports of MPS patients included in the study were evaluated. Echocardiographic examination revealed cardiac pathology in 26 patients (70.2\%). Echocardiographic examination of 11 patients (29.7\%) was normal and the distribution according to MPS types is given in Table 1. The

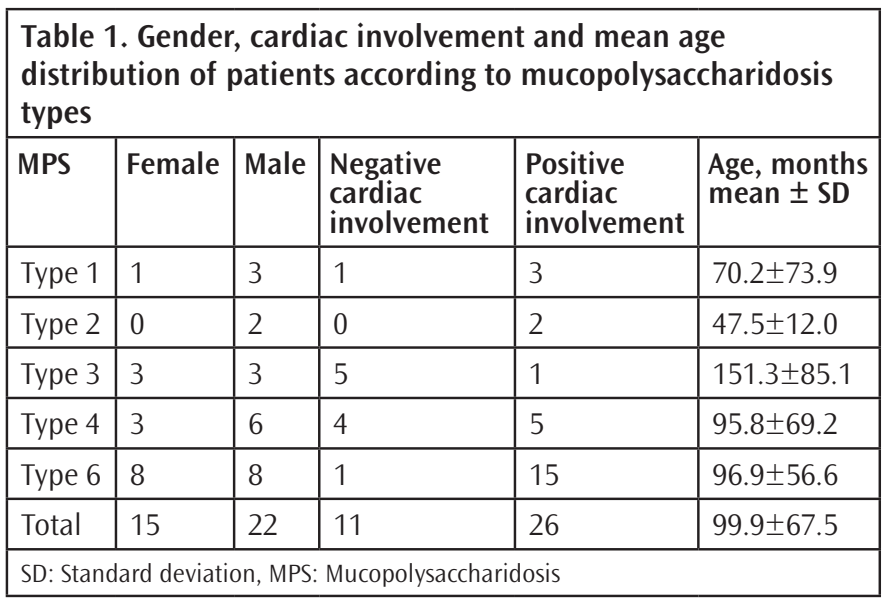

most common echocardiographic findings in type I MPS were mitral valve insufficiency and aortic valve insufficiency. Both type II MPS patients had mitral valve insufficiency. MPS type III patients had the least cardiac pathology, only one patient had bicuspid aortic valve and aortic valve insufficiency, and the remaining five patients had normal echocardiographic examination. The most common pathology in type IV MPS group was aortic valve insufficiency. The echocardiographic findings were most commonly observed in the MPS type VI group, and the most common pathology was mitral valve insufficiency (81.2\%) and aortic valve insufficiency (37.5\%) (Table 2). Regarding echocardiographic findings in all patients, $70.2 \%$ of the patients had cardiac involvement. It was seen that mitral valve was the most affected (59.5\%) structure and the most common pathology was mitral valve insufficiency (51.4\%). The second most common pathology was aortic valve insufficiency (32.4\%). In addition, five patients had cardiomyopathy, three patients had bicuspid aortic valve and two patients had left ventricular hypertrophy.

\section{DISCUSSION}

In MPS patients, accumulation of GAG in the endocardium and myocardium can cause cardiac involvement in all types. Cardiac findings vary from asymptomatic valve involvement to severe left ventricular failure and are a significant cause of mortality. Cardiac involvement is reported to be early and frequent in patients with MPS, especially in types I, II and IV $(3,4)$. Thickening and loss of function of the heart valves (especially in the left heart) and hypertrophy are common, and coronary artery involvement or conduction disorders are rare (4). Electrocardiography and echocardiography are the key

\begin{tabular}{|c|c|c|c|c|c|c|}
\hline Echocardiographic findings & $\begin{array}{l}\text { MPS type I } \\
(n=4)\end{array}$ & $\begin{array}{l}\text { MPS type II } \\
(\mathrm{n}=2)\end{array}$ & $\begin{array}{l}\text { MPS type III } \\
(n=6)\end{array}$ & $\begin{array}{l}\text { MPS type IV } \\
(n=9)\end{array}$ & $\begin{array}{l}\text { MPS type VI } \\
(n=16)\end{array}$ & Total $(n=37)$ \\
\hline Mitral valve insufficiency & $3(75 \%)$ & $2(100 \%)$ & & $1(11.1 \%)$ & $13(81.2 \%)$ & $19(51.4 \%)$ \\
\hline Mitral valve prolapse & $1(25 \%)$ & & & & & $1(2.7 \%)$ \\
\hline Mitral valve thickening & & & & $1(11.1 \%)$ & & $1(2.7 \%)$ \\
\hline Mitral valve stenosis & & & & & $1(6.3 \%)$ & $1(2.7 \%)$ \\
\hline Aortic valve insufficiency & $2(50 \%)$ & & $1(16.7 \%)$ & $3(33.3 \%)$ & $6(37.5 \%)$ & $12(32.4 \%)$ \\
\hline Aortic valve prolapse & & & & & & - \\
\hline Aortic valve thickening & & & & $1(11.1 \%)$ & & $1(2.7 \%)$ \\
\hline Aortic valvestenosis & & & & & & - \\
\hline Bicuspid aortic valve & & & $1(16.7 \%)$ & $1(11.1 \%)$ & $1(6.3 \%)$ & $3(8.1 \%)$ \\
\hline Left ventricular hypertrophy & & & & & $2(12.5 \%)$ & $2(5.4 \%)$ \\
\hline Tricuspid valve insufficiency & & & & $1(11.1 \%)$ & $1(6.3 \%)$ & $2(5.4 \%)$ \\
\hline Cardiomyopathy & $1(25 \%)$ & $1(50 \%)$ & & & $3(18.8 \%)$ & $5(13.5 \%)$ \\
\hline
\end{tabular}


tests to evaluate the cardiac involvement (4). However, it should be kept in mind that deformities (chest wall deformities) due to skeletal system involvement may cause difficulties during echocardiography examination in these patients. In our study, echocardiography reports of 37 MPS patients were examined. The reports of 11 patients were normal and 26 patients (70.2\%) had cardiac involvement. The most frequent involvement was in the mitral valve (59.5\%). The most common pathology was mitral valve insufficiency (51.4\%), followed by aortic valve insufficiency (32.4\%).

MPS type IV group had the highest cardiac involvement and MPS type III group had the lowest. In addition, five patients had cardiomyopathy, three patients had bicuspid aortic valve and two patients had left ventricular hypertrophy. In the literature, Mohan et al. (5) reported cardiac pathologies as mitral regurgitation (29\%), aortic insufficiency (16\%) and mitral stenosis (12\%). It was also emphasized that mitral and aortic valve involvement increased with age (5). In 2010, 26 MPS patients were examined by Leal et al. (6) and the most common cardiac pathologies were mitral valve involvement (60\%), left ventricular failure (43\%), pulmonary hypertension (36\%) and aortic valve involvement (35\%). In the same study, it was reported that the most severe mitral involvement was in patients with MPS type VI (6). In our study, cardiac involvement was more common in MPS type VI patients, because the number of patients with MPS type VI was higher. In a study by Moog et al. (7) in 20 adult MPS type IIIB patients, one patient had mitral regurgitation, one patient had myocardial infarction, three patients had cardiomyopathy and four patients had atrial fibrillation. As a result of their study, cardiac involvement was reported to be low in MPS type III patients, but it was emphasized that cardiac examinations and investigations should not be neglected (7). In our study group, similarly, MPS type III patients had the least cardiac involvement. In the study of Braunlin et al. (8) it was reported that left ventricular hypertrophy improved in MPS type I patients after enzyme replacement therapy, but that mitral and aortic valve pathologies were not improved. In the study of Fesslová et al. (9) it was reported that cardiac functions might worsen immediately after bone marrow transplantation, but stabilization was achieved in long-term follow-up.

\section{CONCLUSION}

As a result, cardiac involvement and complications are frequently seen in MPS. Heart failure, coronary artery involvement and arrhythmias are the main causes of mortality. Early diagnosis of MPS and initiation of enzyme replacement therapy may improve cardiac involvement. In general, it should be noted that valve involvement may be progressive and may require surgical intervention over time. For all these reasons, cardiac evaluation should be performed regularly in MPS patients at least once a year, accompanied by electrocardiogram and echocardiography.

\section{Ethics}

Ethics Committee Approval: The study was approved by the İstanbul Okmeydanı Training and Research Hospital Ethics Committee (approval number: 48670771-514.10).

Informed Consent: Informed consent from was patient.

Peer-review: Externally peer-reviewed.

\section{Authorship Contributions}

Surgical and Medical Practices: N.E.C.., M.K., Concept: N.E.Ç., Design: N.E.C.., Data Collection or Processing: N.E.C.., M.K., Analysis or Interpretation: N.E.C.., M.K., Literature Search: N.E.C,., M.K., Writing: N.E.Ç., M.K.

Conflict of Interest: No conflict of interest was declared by the authors.

Financial Disclosure: The authors declared that this study received no financial support.

\section{REFERENCES}

1. Stapleton M, Arunkumar N, Kubaski F, Mason RW, Tadao O, Tomatsu S. Clinical presentation and diagnosis of mucopolysaccharidoses. Mol Genet Metab 2018;125:4-17.

2. Fernandes J, Saudubray J-M, Berghe G van den, Walter JH, editors. Inborn Metabolic Diseases: Diagnosis and Treatment. 4th ed. Berlin Heidelberg: Springer-Verlag 2006:580-3.

3. Dangel JH. Cardiovascular changes in children with mucopolysaccharide storage diseases and related disorders--clinical and echocardiographic findings in 64 patients. Eur J Pediatr 1998;157:534-8.

4. Braunlin EA, Harmatz PR, Scarpa M, Furlanetto B, Kampmann C, Loehr JP, et al. Cardiac disease in patients with mucopolysaccharidosis: presentation, diagnosis and management. J Inherit Metab Dis 2011;34:1183-97.

5. Mohan UR, Hay AA, Cleary MA, Wraith JE, Patel RG. Cardiovascular changes in children with mucopolysaccharide disorders. Acta Paediatr 2002;91:799804.

6. Leal GN, de Paula AC, Leone C, Kim CA. Echocardiographic study of paediatric patients with mucopolysaccharidosis. Cardiol Young 2010;20:254-61.

7. Moog U, van Mierlo I, van Schrojenstein Lantman-de Valk HMJ, Spaapen L, Maaskant MA, Curfs LMG. Is Sanfilippo type B in your mind when you see adults with mental retardation and behavioral problems? Am J Med Genet C Semin Med Genet 2007 15;145:293-301.

8. Braunlin EA, Berry JM, Whitley CB. Cardiac findings after enzyme replacement therapy for mucopolysaccharidosis type I. Am J Cardiol 2006;98:416-8.

9. Fesslová V, Corti P, Sersale G, Rovelli A, Russo P, Mannarino S, et al. The natural course and the impact of therapies of cardiac involvement in the mucopolysaccharidoses. Cardiol Young 2009;19:170-8. 